\title{
Binary Linear Codes and Binary Matrices
}

\author{
Driss Harzalla Al \\ University Chouaib Doukkali, Faculty of Sciences \\ Department of Mathematics, EI Jadida, Morocco \\ 68harzalla@gmail.com
}

\begin{abstract}
In [1, the automorphism group of a binary linear code is computed by identifying it with one of its optimal generator matrices. This identification is effective for the computation of the automorphism group of a binary linear code. As application, we show in this work that the automorphism group of the binary triple-error-correcting primitive $B C H$ code $C$ of length $n=31$ is the general linear group $G L_{5}\left(F_{2}\right)$ on the vector space $F_{32}$ over $F_{2}$. Numerical examples are given by the use of some theoretical software : GAP 4.8.5 (Group Algorithm Programming), Q-extension and Grin 4.0 (Graph Interface).
\end{abstract}

Keywords: Automorphism, Binary matrix, Bipartite graph, Optimal generator matrix, Binary BCH codes.

\section{Introduction}

A linear $[n, k]$-code $C$ over $F_{2}$ is a $k$-dimensional subspace of the vector space $\left(F_{2}\right)^{n}$. Let $\operatorname{Cod}_{n}$ be the set of all binary linear codes of dimension $k$ and of fixed length $n$, the natural action of the symmetric group $S_{n}$ on $C_{0} d_{n}$ is defined by: $\sigma(C)=\left\{\sigma\left(\left(c_{i}\right)_{i}\right)=\left(c_{\sigma(i)}\right)_{i} / c \in C\right\}$. We say that $C_{1}$ and $C_{2}$ are isomorphic or equivalent and we write $C_{1} \cong C_{2}$ if and only if there exist $\sigma \in S_{n}$ such that $\sigma\left(C_{1}\right)=C_{2}, \sigma$ is said to be an isomorphism and in case $C_{1}=C_{2}$, $\sigma$ is said to be an automorphism. We deduce from the above that for fixed $n, \operatorname{Aut}(C)=\left\{\sigma \in S_{n} / \sigma(C)=C\right\}$ is the stabilizer $S_{t a b} b_{n}(C)$ under the above natural action and $A u t(C)$ is then a subgroup of $S_{n}$ called the automorphism group of $C$.

It is shown in [1] that a binary linear code and a binary matrix represent the same object in the sense that they have the same automorphism group up to an isomorphism. The determination of the structure of codes, computation of their weight distribution, classification of codes, decoding algorithm and cryptography, all these problems can be relatively simplified by the use of the notion of the automorphism group. In the general case the determination of automorphism group of linear codes and that of graphs is known to be a difficult problem.

A generator matrix $G$ for a linear $[n, k b b b]-\operatorname{code} C$ is a $(k \times n)$-matrix whose rows form a basis for $C$, and its parity check matrix $H$ is an $((n-k) \times n)$-matrix with property $G H^{T}=0$ where 0 is the $k \times(n-k)$ null matrix.

Recall that the Hamming distance $d\left(m, m^{\prime}\right)$ between two code-words of a binary $[n, k]$-code $C$ is the number of positions by which the two code-words differ. The Hamming weight $w t(m)$ of a code-word $m \in C$ is $d(m, 0)$. A linear $[n, k, d]$-code $C$ over $F_{2}$ is a $k$-dimensional subspace of the vector space $\left(F_{2}\right)^{n}$, where

$$
d=d(C)=\min _{m \neq m^{\prime} \in C} d\left(m, m^{\prime}\right)=\min _{0 \neq m \in C} w t(m)
$$

Let $M_{k \times n}$ be the set of all $k \times n$ binary matrices with $k<n$ and let $A, B \in M_{k \times n}$. We define a natural action of $S_{n}$ on $M_{k \times n}$ as follows: if $A=\left[c_{1}, c_{2}, \ldots, c_{n}\right]$ and $\sigma \in S_{n}$ then, $A \sigma=\left[c_{\sigma(1)}, c_{\sigma(2)}, \ldots, c_{\sigma(n)}\right]$. We say that $A$ and $B$ are isomorphic and we write $A \cong B$ if there exist $\sigma \in S_{n}$ such that the set Rows $(A \sigma)$ of rows of $A \sigma$ is equal to the set $\operatorname{Rows}(B)$ of rows of $B$. $\sigma$ is said to be an isomorphism and in case $A=B, \sigma$ is said to be an automorphism. The set

$$
\operatorname{Aut}(A)=\left\{\sigma \in S_{n} / \operatorname{Rows}(A \sigma)=\operatorname{Rows}(A)\right\}
$$

\footnotetext{
${ }^{*}$ Corresponding author.Driss Harzalla 68harzalla@gmail.com
} 2010 Mathematics Subject Classification: 94B05, $11 \mathrm{~T} 71$. 
is called the automorphism group of the binary matrix $A$ which is a subgroup of $S_{n}$. We associate with the generator matrix $G$ a binary matrix $B[G]$ as described in the following algorithm [1]:

The algorithm: we begin with the first row vector $r_{1}$ of $G$ and we search all the code-words of $C$ different from $r_{1}$ and having the same weight as $r_{1}$. If they exist they are all added at the beginning of $G$ as row vectors, else we move to the second row vector $r_{2}$ of $G$ and we repeat the same procedure as before. We repeat this process until exhaustion of all row vectors of $G$. This process ends and the binary matrix $B[G]$ is constructed (see $[1$, Theorem 2.1$]$ ).

Theorem: The code $C$ and the binary matrix $B[G]$ have the same automorphism group: $A u t(C)=A u t(B[G])$

Corollary: If $G_{1}$ and $G_{2}$ are two generator matrices of the code $C$ then the corresponding binary matrices $B\left[G_{1}\right]$ and $B\left[G_{2}\right]$ have the same automorphism group.

Definition: A generator matrix $O G$ is said to be an optimal generator matrix of an $[n, k]$-code if we have

$O G \in \underset{G \in \operatorname{Gen}(C)}{\arg \min }\left(\sum_{i=1}^{i=k} A_{w_{g_{i}}}\right)$ where $\operatorname{Gen}(C)$ is the set of all generator matrices $G$ of the code $C$ and $\left(A_{w_{g_{i}}}\right)_{0 \leq i \leq n}$, $w_{g_{i}}=w t\left(g_{i}\right)$ the weight distribution of rows $g_{i}$ of $G$. The associated binary matrix $O B[G]$ is also said to be optimal.

We recall that binary Bose-Chaudhuri-Hockenghem code or binary BCH code for short is the largest possible cyclic code of length $n$ over the field $F_{2}$, whose generator polynomial has zeros $a^{b}, a^{b+1}, a^{b+1}, \ldots, a^{b+\delta-2}$ where $a$ is a primitive $n^{t h}$ root of unity in the splitting field $F_{2^{m}}, b$ is an integer $0 \leq b \leq n-\delta+1$ and $m$ is the multiplicative order of 2 modulo $\mathrm{n}$. The length $\mathrm{n}$ of the code and the size $q=2$ of the field must be relatively prime. The generator polynomial is equal to the least common multiple of the minimal polynomials of $a^{b}, a^{b+1}, a^{b+1}, \ldots, a^{b+\delta-2}$. Special cases are $b=1$ (resulting codes are called narrow-sense BCH codes), and $n=2^{m}-1$ (known as primitive BCH codes). The largest value of $d$ for which the $\mathrm{BCH}$ code with designed distance $d$ coincides with the BCH code with designed distance $\delta$ is called the Bose distance of the code. The true minimum distance of the code is greater than or equal to the Bose distance [3].

\section{Application to binary $\mathrm{BCH}$ Code of parameters $n=31, b=1, \delta=7$}

The weight distribution and the minimum distance of the binary narrow-sense primitive $\mathrm{BCH}$ code $C=B C H C o d e(n=$ $31, b=1, \delta=5, G F(2))$ of parameters $n=31, b=1, \delta=5$ are given in the following GAP program

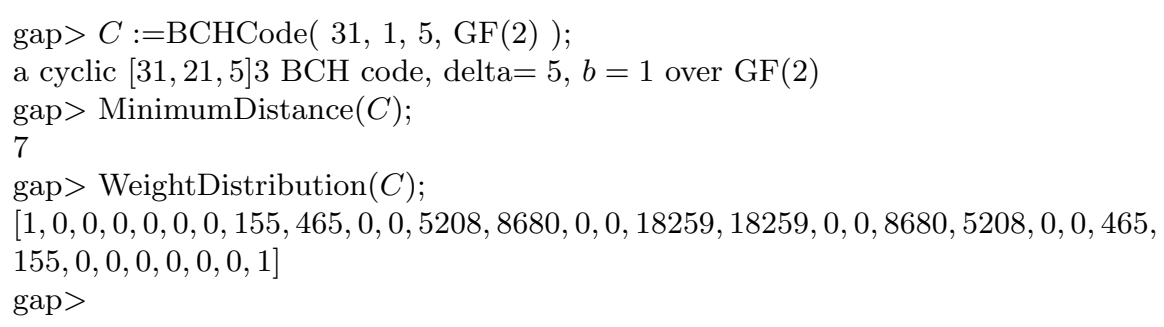


This code is a cyclic $[31,16,7]$-code of minimum distance 7 , dimension 16 , length 31 and its weight distribution is: $[1,0,0,0,0,0,0,155,465,0,0,5208,8680,0,0,18259,18259,0,0,8680,5208,0,0,465,155,0,0,0,0,0,0,1]$ By using the GAP command MinimumWeightWords(C), one can look for a generator matrix among the 155 words of minimal weight. And by using Q-Extension tools [2] the following optimal generator matrix is then obtained.

$$
O G=\left(\begin{array}{c}
0000000000010000000010101101001 \\
0000000000011100100000100000011 \\
0000000000100010001010011000001 \\
0000000000100000000101011010010 \\
0000000000111001000001000000110 \\
0000000001000100010100110000010 \\
0000000001000000001010110100100 \\
0000000010001000101001100000100 \\
0000000100010001010011000001000 \\
0000001000100000101001000000011 \\
0000010001000001010010000000110 \\
0000100010000010100100000001100 \\
0001000101001100000100000000001 \\
0010001010011000001000000000010 \\
0100010100110000010000000000100 \\
1000101001100000100000000001000
\end{array}\right)
$$


The optimal binary matrix associated with the code $C$ is the binary $(155 \times 31)$ - matrix of rows


000001000000110000000 0001000101001100000100000000001 0001000100000101001000000011000 0001000100010100110000010000000 0001000001100000001000000101001 0001000001010010000000110000001 0001000000110000000000011100100 0001000000101001000100000110000 0001000000000010001000101001100 0001000000001010110100100000000 0001010110100100000000000100000 0001010010000000110000001000100 0001010010001000001100000001000 0001010011000001000000000010001 0001100000001000000101001000100 0001100000000000111001000001000 0001100000010001000001010010000 0001110010000010000001100000000 0010001010011000001000000000010 0010001000001100000001000000101 0010001000001010010000000110000 0010001000101001100000100000000 0010000011000000010000001010010 0010000010100100000001100000010 0010000010000001100000000000111 0010000001100000000000111001000 0010000001010010001000001100000 001000000110000001000100000101 0010000000000000101 0010000000000010000000010101101 0010000000010010001010011000 01000101001000000000000 0010100100000011000001000000 0010100100000001100000010001000 00101001000100001100000010000 001010011000001000000000100010 0011000000010000001010010001000 0011000000000001110010000010000 0011000000100010000010100100000 0011000001000000000010001000101 0011100100000100000011000000000 0100010100110000010000000000100 0100010000011000000010000001010 0100010000010100100000001100000 0100010001010011000001000000000 0100000110000000100000010100100 0100000101001000000011000000100 0100000100000011000000000001110 0100000011000000000001110010000 0100000010100100010000011000000 0100000001100000010001000001010 0100000000000100000000101011010

\begin{tabular}{||l||}
\hline 0100000000001000100010100110000 \\
0100000000101011010010000000000 \\
0100110000010000000000100010001 \\
0100100000001100000010001000001 \\
0100100000000000100000000101011 \\
0100100010000011000000010000001 \\
0101011010010000000000010000000 \\
0101001000000011000000100010000 \\
0101001000100000110000000100000 \\
0101001100000100000000001000100 \\
0101101001000000000001000000001 \\
0110000000100000010100100010000 \\
0110000000000011100100000100000 \\
0110000001000100000101001000000 \\
0110000010000000000100010001010 \\
0110100100000000000100000000101 \\
0111001000001000000110000000000 \\
1000101001100000100000000001000 \\
1000100000110000000100000010100 \\
1000100000101001000000011000000 \\
1000100010100110000010000000000 \\
1000001100000001000000101001000 \\
1000001010010000000110000001000 \\
1000001000000000010001000101001 \\
1000001000000110000000000011100 \\
1000000110000000000011100100000 \\
1000000100010000010100100000001 \\
1000000101001000100000110000000 \\
1000000010000001010010001000001 \\
1000000011000000100010000010100 \\
1000000000001110010000010000001 \\
1000000000001000000001010110100 \\
1000000000010001000101001100000 \\
1000000001010110100100000000000 \\
1001100000100000000001000100010 \\
1001000001000000110000000000011 \\
1001000000011000000100010000010 \\
1001000000000001000000001010110 \\
1001000100000110000000100000010 \\
1010110100100000000000100000000 \\
1010010000000000010000000010101 \\
1010010000000110000001000100000 \\
1010010001000001100000001000000 \\
1010011000001000000000010001000 \\
1011010010000000000010000000010 \\
1100100000100000011000000000001 \\
1100000001000000101001000100000 \\
1100000000000111001000001000000 \\
1100000010001000001010010000000 \\
1100000100000000001000100010100 \\
1101001000000000001000000001010 \\
1110010000010000001100000000000 \\
\hline
\end{tabular} \mid

By using Q-Extension tools 2, the set generating the group of the optimal binary matrix is obtained. Then, the GAP command GroupWithGenerators $([])$ determines the structure of this group as indicated in the next two programs. 
- Q-extension program:

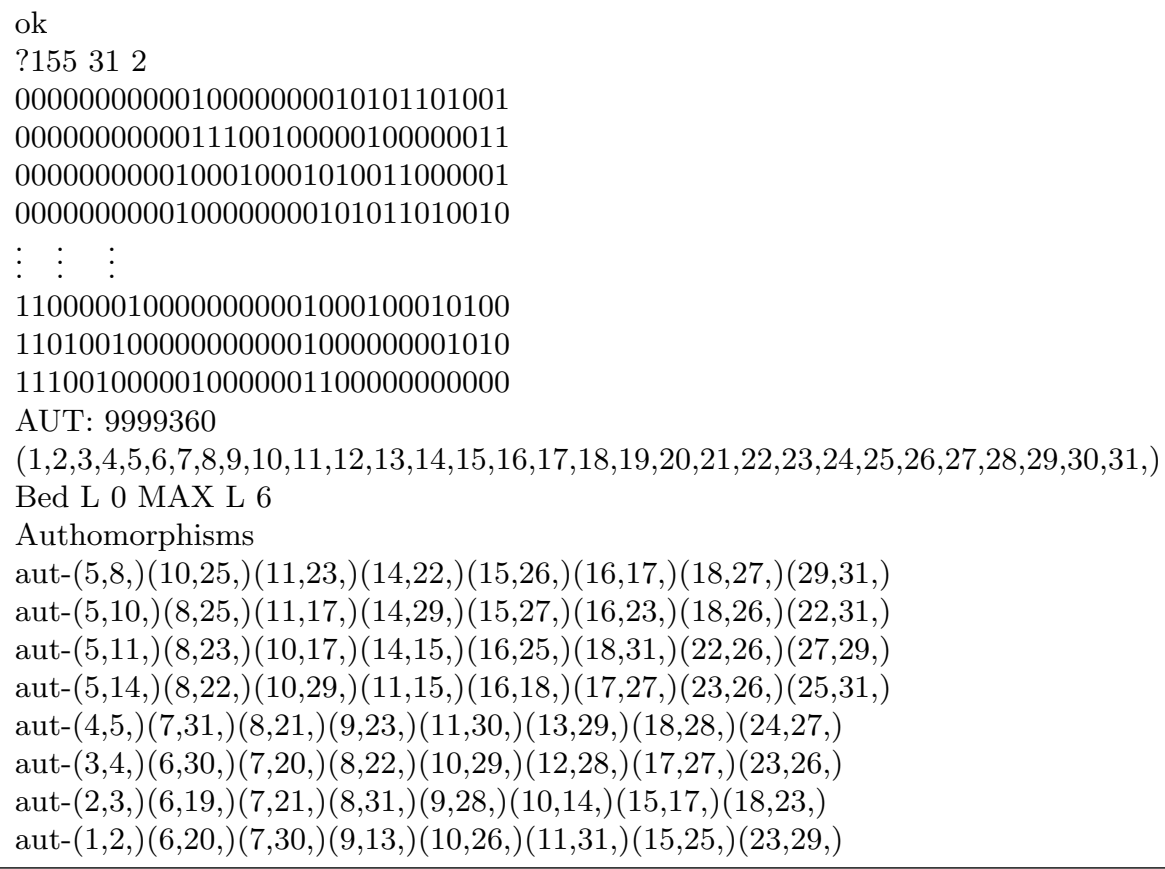

- GAP (GUAVA) program:

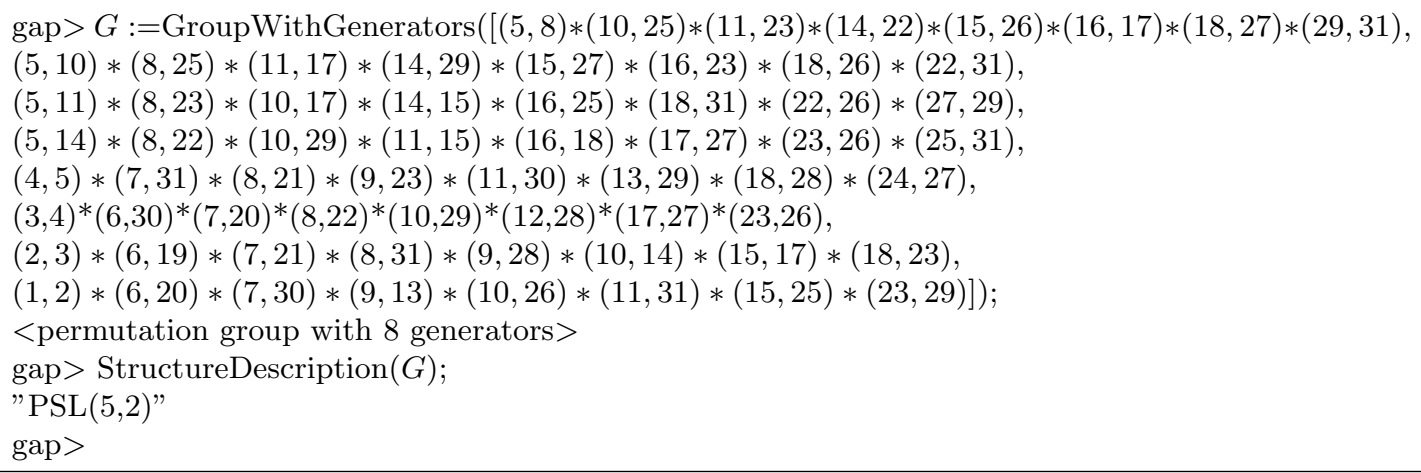

We deduce that the automorphism group of the the binary narrow-sense primitive $\mathrm{BCH}$ code $C=B C H C$ ode $(n=$ $31, b=1, \delta=5, G F(2))$ of parameters $n=31, b=1, \delta=5$ is the special projective linear group $P S L(3,2)$. We recall here that $P S L(5,2)=S L(5,2)=G L(5,2)$, the special (general) linear group of $5 \times 5$ matrices over the field with 2 elements.

\section{Conclusion}

In general, let $C$ be an $[n, k]$-code and $O G$ its optimal generator matrix. Let $r_{i}, i=1,2, \ldots, k$ be the rows of $O G$ and $\left(A_{w_{i}}\right), w_{i}=w t\left(r_{i}\right)$ its weight distribution.

Let $O B$ be the optimal binary matrix described in the algorithm 1 . Recall that $A u t(C)$ and $A u t(O B[G])$ are the same.

The method of the optimal binary matrix is effective since in the general case the value of

$$
\frac{\min _{G \in \operatorname{OGen}(C)}\left(\sum_{i=1}^{i=k} A_{w_{i}}\right)}{A_{w_{1}}+A_{w_{2}}+\ldots+A_{w_{k}}}
$$


is very small.

In the example of the binary narrow-sense primitive $\mathrm{BCH}$ code $C=B C H C o d e(n=31, b=1, \delta=5, G F(2))$ of parameters $n=31, b=1, \delta=5$ we have:

$$
\frac{\# \text { Rows }(O B[G])}{\# \text { Rows }([C])}=\frac{155}{2^{16}} \approx 0,0023
$$

The main result of this work is the result given in the following theorem.

Theorem 3.1. The automorphism group of the binary triple-error-correcting primitive $B C H$ code $C$ of length $n=2^{m}-1=31$ with $m=5$, is $G L_{5}\left(F_{2}\right)$, where $G L_{5}\left(F_{2}\right)$ is the general linear group on the vector space $F_{32}$ over $F_{2}$.

ACKNOWLEDGEMENTS.

The author gratefully acknowledge the referees for their useful suggestions and comments.

\section{References}

[1] D. Harzalla. Optimal Generator matrix and the automorphism groups of linear binary codes, International Journal of Engineering Issues, Infinity Science, 2:53-61, 2016.

[2] G. Iliya Bouyukliev. What is Q-Extension?, Serdica J. Computing, 1:553-564, 2007.

[3] F. J. MacWilliams and N. J. A. Sloane. The theory of error-correcting codes, Elsevier-North-Holland, Amsterdam, 1977. 